\title{
The pretransplant systemic metabolic profile reflects a risk of acute graft versus host disease after allogeneic stem cell transplantation
}

\author{
Håkon Reikvam ${ }^{1,2} \cdot$ Kimberley Hatfield $^{1}$ - Øystein Bruserud ${ }^{1,2}$
}

Received: 31 May 2015/Accepted: 29 July 2015/Published online: 16 November 2015

(C) European Union 2015

\begin{abstract}
Allogeneic stem cell transplantation is used in the treatment of younger patients with severe hematological diseases, especially hematological malignancies, and acute graft versus host disease (GVHD) is then an important immune-mediated posttransplant complication. Several risk factors for acute GVHD have been identified, including pretransplant factors that possibly influence the posttranspant course through their effects on host immunocompetent cells. Metabolic regulation is important for immunoregulation, and we therefore investigated whether the pretransplant metabolic status of allotransplant recipients was associated with later acute GVHD. In our population-based study we investigated the systemic (serum) metabolic profile for 86 consecutive allotransplant recipients. The samples were collected before start of the pretransplant conditioning therapy. Patients who developed later acute GVHD especially showed altered pretransplant amino acid metabolism, including (1) altered metabolism of immunoregulatory branched chain amino acids (leucine, isoleucine and valine); and (2) altered levels of potentially proinflammatory tyrosine
\end{abstract}

Electronic supplementary material The online version of this article (doi:10.1007/s11306-015-0880-x) contains supplementary material, which is available to authorized users.

Øystein Bruserud

Oystein.Bruserud@helse-bergen.no

Håkon Reikvam

Hakon.Reikvam@med.uib.no

Kimberley Hatfield

Kimberley.Hatfield@med.uib.no

1 Section Hematology, Institute of Clinical Science, University of Bergen, Bergen, Norway

2 Section Hematology, Department of Medicine, Haukeland University Hospital, 5021 Bergen, Norway metabolites ( $\mathrm{p}$-cresol sulphate, 3-phenylpropionate) formed by the gastrointestinal microbial flora. However, isobutyrylcarnitine and propyonylcarnitine levels were also altered; the carnitines are important for the transport of fatty acids and may also be important for the release of immunoregulatory cytokines in allotransplant recipients. These metabolic alterations were associated with an ongoing pretransplant acute phase reaction or early hematopoietic/immune reconstitution. Thus, allotransplant recipients developing acute GVHD showed altered preconditioning/pretransplant levels of several immunoregulatory metabolites. Our hypothesis is that these metabolites alter or activate recipient immunocompetent cells and thereby enhance or initiate anti-recipient immune reactivity.

Keywords Metabolomics - Allogeneic stem cell transplantation - Acute leukemia - Acute graft versus host disease

\section{Introduction}

Allogeneic hematopoietic stem cell transplantation (alloSCT) represents an effective therapeutic strategy in younger patients with a variety of severe hematological disorders. The strong antileukemic effect of allo-SCT is due to the intensive conditioning therapy, nonspecific graft versus host reactivity and specific graft versus leukemia (GVL) effects (Kersey 2010). However, these beneficial effects are counteracted by the risk of severe and potentially lethal posttransplant complications including serious infections, immune-mediated acute and chronic graft-versus-host disease (GVHD) and treatment-related organ toxicity (Gooley et al. 2010). The decision to recommend allo-SCT therefore thus has to be individualized and based 
on the expected long-term disease-free survival with conventional chemotherapy alone versus the risk of relapse and the risk of treatment-related mortality/morbidity after allogeneic transplantation. Several strategies based on pretransplant prognostic factors of long-term survival have been suggested (Gratwohl 2012; Servais et al. 2014; Sorror 2009). However, these systems are mainly based on clinical factors and may be improved if additional biological factors could be incorporated, e.g. the pretransplant serum profiles of immunoregulatory cytokines that differ between allotransplant recipients with and without posttransplant acute GVHD (Reikvam et al. 2012a, b, 2013).

Metabolomic profiling, or metabolomics, is the study of low molecular weight compounds in biological fluids and metabolites can then be defined as non-peptide molecules often representing end products of cellular activity. Therefore, changes in metabolite concentrations reveal the range of biochemical effects induced by diseases and/or therapeutic intervention. During the last years metabolomic profiling has become possible through the development of new technologies, and studies in patients with acute myeloid leukemia (AML) have described both a specific metabolic profile associated with mutations in the isocitrate dehydrogenases 1 and 2 genes (IDH 1/2) (Wang et al. 2013) as well as a distinct glucose metabolism signature associated with resistance to cytarabine and decreased survival (Chen et al. 2014).

The overall nutritional/metabolic status as well as the levels of several single metabolites is important for immunoregulation (Tvedt et al. 2016). In this context we have investigated whether pretransplant metabolic profiles reflects a risk of posttranspant acute GVHD, and we describe associations between the pretransplant metabolic profile and the posttransplant clinical course.

\section{Materials and methods}

\subsection{Patients}

The study was approved by the local Ethics Committee (Regional Ethics Committee III, University of Bergen, Norway) and samples collected after written informed consent. The study included 86 consecutively allotransplanted adult patients ( 54 men and 32 women; median age 45 years with range 15-69 years) from a defined geographic area (Norwegian Health Regions III, IV and V) transplanted with a family donor. The decision to perform an allotransplantation was taken by the Norwegian Advisory Board for Stem Cell Transplantation and based on national guidelines. Thus, our study is population-based and includes an unselected and consecutive group of wellcharacterized patients.
The clinical characteristics of the patient are given in Table 1 and Supplementary Fig. 1. Most patients received GVHD prophylaxis with cyclosporine A and methotrexate, only two patients received cyclosporine A alone. Patients were transplanted with granulocyte colony-stimulating factor (G-CSF) mobilized peripheral blood stem cells derived from HLA-matched family donors (aplastic anemia patients received bone marrow grafts). Neutrophil reconstitution was defined as three consecutive days with neutrophil counts of at least $0.2 \times 10^{9} / \mathrm{L}$, and platelet reconstitution as stable platelet counts exceeding $20 \times 10^{9} /$ $\mathrm{L}$ for at least three consecutive days. All patients were carefully examined for and classified with regard to comorbidity according to Sorror et al. (2009); none of the patients had liver or renal disease and the overall comorbidity score was low (1 or 0 ). Sinusoidal obstruction syndrome was not diagnosed in any patient.

All samples were collected before start of conditioning therapy (median 19 days before, range 3-56 days). GVHD was diagnosed according to generally accepted criteria (Filipovich 2008). Briefly, the diagnosis of acute GVHD was generally based on careful clinical evaluation and additional skin biopsies for patients with skin involvement alone. The diagnosis of acute GVHD for the patients with liver and/or gastrointestinal involvement was also based on careful clinical evaluation and additional biopsies (including eight with skin biopsies and five with biopsies from the gastrointestinal tract) except for one patient with liver involvement for whom acute GVHD was a clinical diagnosis.

\subsection{Sample preparation, analysis of metabolite serum levels and bioinformatical analyses}

All venous blood samples were collected into sterile plastic tubes (BD Vacutainer ${ }^{\circledR} \mathrm{SST}^{\mathrm{TM}}$ Serum Separation Tubes, Becton-Dickenson; Franklin Lakes, NJ, USA) and allowed to coagulate for $120 \mathrm{~min}$ at room temperature $\left(18^{\circ} \mathrm{C}\right)$ before centrifugation ( $300 \mathrm{~g}$ for $10 \mathrm{~min}$ ) and serum collection. All samples were immediately frozen at $-70{ }^{\circ} \mathrm{C}$ until analyzed.

Metabolomic profiling analysis of all samples was carried out in collaboration with Metabolon ${ }^{\circledR}$ as described previously (Evans et al. 2014). Each sample was accessioned into the Metabolon LIMS system and was assigned by the LIMS a unique identifier, and was used to track all sample handling, tasks, results, etc. The samples (and all derived aliquots) were tracked by the LIMS system. Samples were prepared using the automated MicroLab STAR ${ }^{\circledR}$ system from Hamilton Company. A recovery standard was added prior to the first step in the extraction process for QC purposes. To remove protein, dissociate small molecules bound to protein or trapped in the precipitated protein 
Table 1 Demographical, clinical and laboratory data for the 86 patients included in the study

\begin{tabular}{|c|c|}
\hline Patient characteristics & Observation \\
\hline \multicolumn{2}{|l|}{ Demographic data and disease history } \\
\hline \multicolumn{2}{|l|}{ Gender (numbers) } \\
\hline Male/female & $54 / 32$ \\
\hline Age (years, median and range) & $45(15-69)$ \\
\hline Height (cm, median and range) & $176(149-197)$ \\
\hline Weight (kg, median and range) & $71(42-133)$ \\
\hline BMI $\left(\mathrm{kg} / \mathrm{m}^{2}\right.$, median and range $)$ & $23.3(16.6-39.7)$ \\
\hline \multicolumn{2}{|l|}{ Diagnosis (numbers) } \\
\hline AML & 38 \\
\hline MDS & 16 \\
\hline ALL & 19 \\
\hline CML & 5 \\
\hline CMML & 2 \\
\hline CLL & 1 \\
\hline PMF & 2 \\
\hline AA & 3 \\
\hline \multicolumn{2}{|l|}{ Disease status (numbers) } \\
\hline CR1 & 51 \\
\hline CR2 & 15 \\
\hline No remission & 20 \\
\hline \multicolumn{2}{|l|}{ Conditioning regimen (numbers) } \\
\hline $\mathrm{Bu}+\mathrm{Cy}$ & 69 \\
\hline $\mathrm{ATG}+\mathrm{Cy}$ & 4 \\
\hline $\mathrm{TBI}+\mathrm{Cy}$ & 2 \\
\hline TBI + Eto & 1 \\
\hline $\mathrm{Flu}+\mathrm{Bu}$ & 9 \\
\hline $\mathrm{Flu}+\mathrm{Cy}$ & 1 \\
\hline Acute GVHD (numbers) & 31 out of 77 evaluable patients \\
\hline \multicolumn{2}{|l|}{ Reconstitution (day posttransplant) } \\
\hline \multicolumn{2}{|l|}{ Neutrophils (day; median and range) } \\
\hline$>0.2 \times 10^{9} / \mathrm{L}$ ( 3 consecutive days $)$ & $16(6-52)$ \\
\hline \multicolumn{2}{|l|}{ Platelets (day; median and range) } \\
\hline$>20 \times 10^{9} / \mathrm{L}$ ( 3 consecutive days $)$ & $15(9-33)$ \\
\hline \multicolumn{2}{|l|}{ Baseline pretransplant status } \\
\hline WBC (median and range) & $3.7(0.5-14.1) \times 10^{9} / \mathrm{L}$ \\
\hline $\mathrm{Hb}$ (median and range) & $10.4(7.8-14.1) \mathrm{g} / \mathrm{dL}$ \\
\hline Platelets (median and range) & $141(7-721) \times 10^{9} / \mathrm{L}$ \\
\hline CRP (median and range) & $5(1-120) \mathrm{mg} / \mathrm{L}$ \\
\hline LDH (median and range) & $187(92-1655) \mathrm{UI} / \mathrm{dL}$ \\
\hline
\end{tabular}

Values are unless otherwise stated given as median and range in parenthesis. Height and weight were registered at the start of conditioning therapy

$B M I$ body mass index, $A M L$ acute myelogenous leukemia, $M D S$ myelodysplastic syndrome, $A L L$ acute lymphoblastic leukemia, $C M L$ chronic myelogneous leukemia, $C M M L$ chronic myelomonocytic leukemia, $C L L$ chronic lymfocytic leukemia, $P M F$ primary myelofibrosis, $A A$ aplastic anemia, $C R$ complete remission, $B u$ busulphan, $C y$ cyclophosphamide, $A T G$ anti-thymoglobulin, $T B I$ total body irradiation, Eto etoposide, $F l u$ fludarabine, $G V H D$ graft versus host disease, $W B C$ white blood cell count, $H b$ hemoglobin, $C R P$ C-reactive protein, $L D H$ lactate dehydrogenase matrix, and to recover chemically diverse metabolites, proteins were precipitated with methanol under vigorous shaking for $2 \mathrm{~min}$, followed by centrifugation. The resulting extract was divided into four fractions: one for analysis by UPLC-MS/MS with positive ion mode electrospray ionization, one for analysis by UPLC-MS/MS with 
negative ion mode electrospray ionization, one for analysis by GC-MS, and one sample was reserved for backup. Samples were placed briefly on a TurboVap ${ }^{\circledR}$ (Zymark) to remove the organic solvent. For LC, the samples were stored overnight under nitrogen before preparation for analysis. For GC, each sample was dried under vacuum overnight before preparation for analysis.

Bioinformatical analyses were performed using the J-Express (MolMine AS, Bergen, Norway) (Stavrum et al. 2008). For hierarchical clustering all values were median variance standardized and $\log (2)$ transformed. The complete linkage was used as linkage method, and for distance measured the Pearson correlation was used. Statistical analyses were performed using the Statistical Package for the Social Sciences (SPSS) version 15.0 (SPSS Inc., Chicago, IL, USA). The Chi Square test was used to compare different groups. Unless otherwise stated $p$ values $<0.05$ were regarded as statistically significant.

\section{Results and discussion}

\subsection{Allotransplant recipients are heterogeneous with regard to the pretransplant serum metabolite profile}

Our study included 86 consecutive patients. Nine of these patients (6 men and 3 women, median age 44 years with range 25-56 years) died from early non-GVHD complications (i.e. septicemia, organ toxicity) that developed within 14 days posttransplantation and later progressed to irreversible multiorgan failure. Seven of these patients had AML (one secondary to myelodysplastic syndrome); they were all in first complete remission prior to transplantation except for one patient who was in second complete remission. The two last patients had T-ALL and aplastic anemia, respectively. All patients received myeloablative conditioning treatment, 7 of them with busulfan plus cyclophosphamide. Only three of these patients showed signs of hematopoietic reconstitution at the time of death, and 8 of them died before day +25 posttransplant (median survival 21 days after transplantation, range 7-43 days). Death due to severe septicemia was seen only for one patient (pseudomonas aeruginosa, death on day +7 ). Thus, these patients did not differ from the other patients with regard to these clinical characteristics. The nine patients were not included in our comparison of patients with and without acute GVHD because (1) multiorgan failure developed before acute GVHD would usually be expected, and (2) the complexity of the clinical situation made it impossible to diagnose acute GVHD according to generally accepted criteria but it should be emphasized that none of the patients showed any of the typical symptoms/signs consistent with acute GVHD in the skin, liver or gastrointestinal tract.

A total of 766 metabolites were analyzed in the pretransplant serum samples for all 86 patients (Supplementary Table 1). These metabolites could be divided into the eight main categories (corresponding number of metabolites) amino acids (156), peptides (92), carbohydrates (24), energy metabolism (9), lipids (298), nucleotides (36), cofactors/vitamins (27) and xenobiotics (124). These metabolites represent a broad evaluation of the major metabolic pathways in the cell (Supplementary Table 1).

We first compared the pretransplant metabolic profile of the 31 patients with and the 46 patients without acute GVHD; both principal component analysis and hierarchical clustering then failed to distinguish between these two patient subsets (data not shown). These observations probably reflect the metabolic heterogeneity of our patients when comparing the overall serum metabolite profiles.

\subsection{Patients developing acute GVHD have altered pretransplant levels of several immunoregulatory metabolites}

We then performed a random forest analysis of our patients; this is a supervised classification statistical method that can be used for biomarker identification. By using this methodological approach we could identify metabolic biomarkers that distinguished between patients with and without acute GVHD with a predictive accuracy of $71.5 \%$ (Fig. 1). This number is thus greater than the random chance alone $(\sim 50 \%)$, suggesting that the identified metabolites are candidate biomarkers for predicting risk of GVHD. The figure shows the top 30 metabolites based on the importance for the separation of the two groups. A majority of these metabolites (14 out of 30) are involved in amino acid metabolism, especially the metabolism of immunoregulatory branched chain amino acids (BCAA), but other immunomodulatory metabolites were also identified:

- p-cresol sulphate (de Loor et al. 2005) and 3-phenylpropionate (Nordlund et al. 2012) are tyrosine derivatives that can be formed by the intestinal bacterial flora and later be absorbed. p-cresol sulphate seems to have both proinflammatory and proapoptotic effects (de Loor et al. 2005).

- Isobutyrylcarnitine and propyonylcarnitine levels were also altered; the carnitines are important for the transport of fatty acids and may also be important for the release of immunoregulatory cytokines in allotransplant recipients (Zatarain and Savani 2012).

- Propionylcarnitine have effects on endothelial cells and may thereby influence the trafficking of immunocompetent cells (Andreozzi 2009; Malaguarnera 2012). 


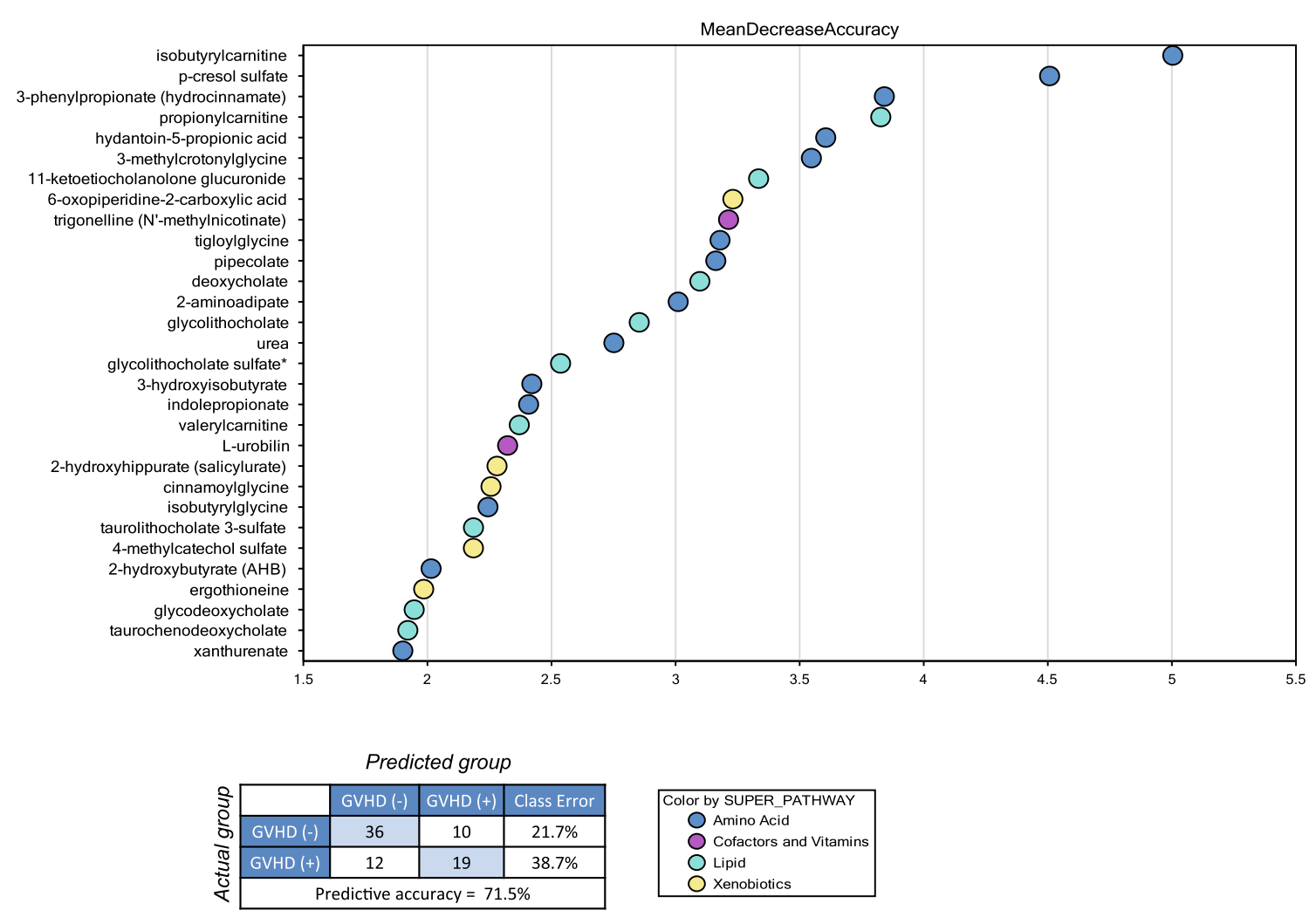

Fig. 1 Random forest analysis of pretransplant metabolite levels; identification of metabolites showing increased serum levels in patients with posttransplant acute GVHD. Random forest analysis could distinguish between the metabolic signatures of patients with and without acute GVHD with a predictive accuracy of $71.5 \%$; this

- Hydantoin-5-propionic acid, the altered level of this metabolite is consistent with altered metabolism of valine and/or isoleucine. Furthermore, 3-methylcrotonylglycine is formed by the metabolism of BCAAs and seems to disrupt mitochondrial energy homeostasis through effects on the respiratory chain, but there is also altered formation of free radicals (Moura et al. 2012).

Thus, altered pretransplant metabolism of immunoregulatory amino acids and/or altered pretransplant levels of immunoregulatory metabolites seem to reflect an increased risk GVHD.

\subsection{Acute GVHD patients show altered pretransplant metabolism of immunoregulatory branched chain amino acids}

We also performed an additional pathway enrichment analysis based on the number of altered metabolites relative to the total number of all analyzed metabolites in a metabolic pathway; this was compared with the total number of altered metabolites relative to all the metabolites analyzed. We identified several metabolic pathways that were enriched in the 31 number is higher than random chance alone $(\sim 50 \%)$ and suggests that these metabolites are candidate biomarkers for increased risk of acute GVHD. The figure presents the top 30 metabolites based on importance to separate the two patient groups

patients with acute GVHD compared with the 46 patients without this posttransplant complication (Fig. 2, Supplementary Table 1), and this alternative analysis showed several similarities to the previous random forest analysis. Firstly, the metabolism of branched chain amino acids (i.e. leucine, isoleucine and valine) differed between patients with and without later acute GVHD. Secondly, tyrosine metabolism was also found to be altered; this observation together with the observed differences in tryptophan, lysine, phenylalanine, and secondary bile acid metabolism suggest that the pretransplant gut function/microbiome is altered in patients who develop acute GVHD (all 86 patients included in the analysis). Thirdly, tryptophan metabolism can also be important in immunoregulation, but even though this term was also increased tryptophan metabolism seems to be less important for the risk of acute GVHD than BCAA metabolism.

We further analyzed the metabolites belonging to the top five ranked enriched metabolic pathways: (1) fatty acid/BCAA metabolism; (2) leucine, isoleucine and valine metabolism; (3) fatty acid synthesis; (4) guannidino and acetamido metabolism; and (5) inositol metabolites. We next performed a hierarchical clustering analysis (Pearson's Correlation, complete linkage) based 
Fig. 2 Pathway enrichment analysis of metabolic profiles associated with acute GVHD. The pathway enrichment analysis was used to identify pathways that were altered in pretransplant samples for patients with later acute GVHD compared to patients without acute GVHD. A pathway enrichment value greater than 1 indicates that the pathway was increased in acute GVHD patients. The top ranked metabolic pathways $(p<0.05$, enrichment value $>2$ ) identified by this comparison $(\mathrm{GVHD}(+)$ versus $\mathrm{GVHD}(-)$ patients) are given in the figure
GVHD (+) / GVHD (-)

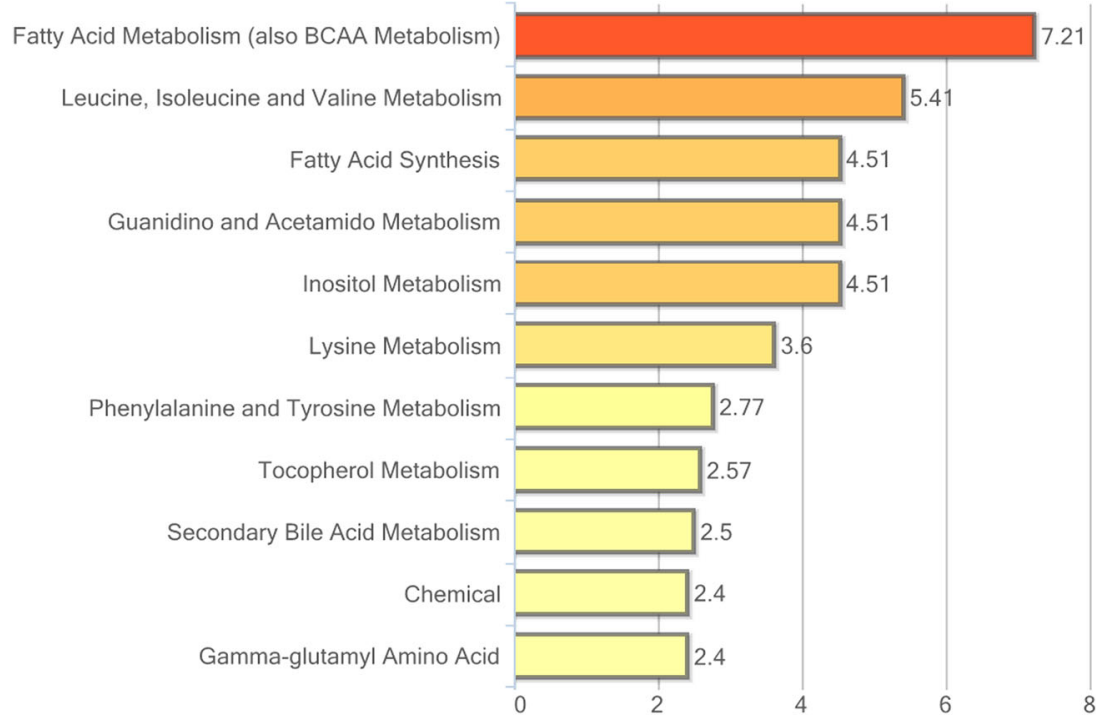

on the 44 metabolites that belonged to these five terms (Fig. 3). There was very little overlap between the five enriched metabolic pathways, and a major part of these metabolites were included in the term leucine, isoleucine and valine metabolism ( 31 of the 44 metabolites). Based on the cluster analysis we were able to identify two main patient clusters; for one cluster 19 out of 34 patients developed acute GVHD whereas for the other cluster only 11 out 52 developed GVHD. This difference was statistically significant (Chi Square test, $\chi^{2}=12.69$, $p=0.0004)$.

We also included the nine patients with early irreversible multiorgan failure in the final clustering analysis; these patients seem to be a heterogeneous subset and the distribution of these patients did not differ between the two main subsets. These nine patients also showed a wide variation in the serum levels of individual metabolites associated with acute GVHD (Fig. 1) and with no differences from the other patients. Taken together these observations suggest that these patients with early death do not represent a group of patients dying from hyperacute GVHD. Other causes of multiorgan failure (i.e. infections, treatment toxicity) seem to be more likely.

\subsection{The metabolic profile associated with an ongoing pretransplant acute phase reaction differs from the acute GVHD profile}

We then investigated how the pretransplant metabolic profile differed between patients with and without signs of ongoing inflammation; we then used the serum level of C-reactive protein (CRP) as a marker for an acute phase reaction and compared patients with $\mathrm{CRP}<10 \mathrm{mg} / \mathrm{L}$ (no inflammation, 58 patients) and $\mathrm{CRP}>10 \mathrm{mg} / \mathrm{L}$ (with inflammation, 28 patients). An initial pathway enrichment analysis identified several metabolic pathways that differed between these two patient subsets (Fig. 4, Supplementary Table 1); 15 terms with a pathway enrichment value exceeding 2 were identified but these terms differed from the acute GVHD analysis (see above) and did not include the branched chain amino acid terms. Furthermore, when doing a random forest analysis the 30 highest ranked metabolites included only 6 amino acid metabolites, 8 lipid metabolites were included together with 4 carbohydrates, 5 nucleotides and 7 peptide metabolites. Thus, these methodological approaches identified other term/metabolites that differed from the acute GVHD-associated profile, suggesting that the association between metabolic profile and acute GVHD is not caused by a pretransplant inflammation/acute phase reaction.

We finally performed a hierarchical clustering analysis based on the results from the analyses (Pearson's Correlation, complete linkage, Fig. 5). Based on the cluster analysis we identified two main patient clusters: one main cluster that included most patients with increased CRP levels and another including most patients with normal/low CRP levels; this difference was highly significant (Chi Square test, $\left.\chi^{2}=8.02, p=0.0046\right)$.

\subsection{Patients with late hematological reconstitution have a metabolomic profile characterized by altered purine and pyrimidine metabolism}

None of the patients showed primary graft failure, and we defined early reconstitution as three consecutive days with 


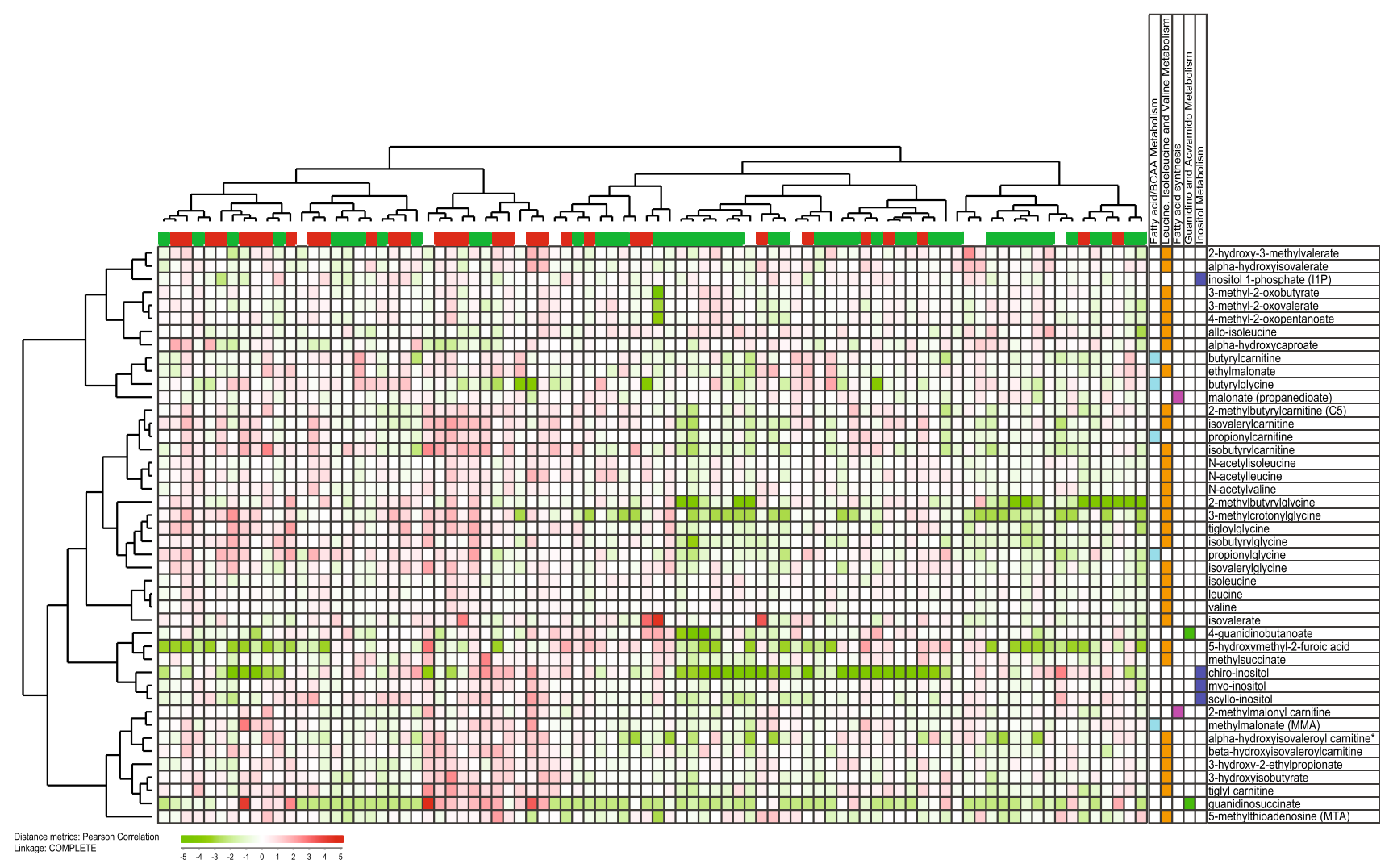

Fig. 3 Hierarchical clustering analysis identified two patient subset highly associated to GVHD. We performed a hierarchical clustering analysis (Pearsons Correaltion, complete linkage) based on the 44 metabolites included in the top five ranked terms from the pathway enrichment analysis presented in Fig. 2. Based on the 44 metabolites belonging to these five terms, we performed a hierarchical clustering analysis. The heat map and according dendrograms are shown in the figure. Red indicates high value and green low value, and the five metabolic pathways can be seen in the left part of the figure. We identified two main clusters; the left main cluster includes a major part of patients who later developed acute GVHD, whereas the right cluster included mainly patients without posttransplant acute GVHD. The frequency of acute GVHD patients differed significantly between the clusters (Chi Square test, $\chi^{2}=12.69, p=0.0004$ )

not change during the study period. Thus, the patients represent a well-defined and relatively homogeneous group of allotransplant recipients.

As explained above we excluded nine patients who died early without the classical diagnostic signs of acute GVHD, but for all except one patient with septicemia the cause of the multiorgan failure could not be identified. One possible cause is treatment toxicity, another possibility being hyperacute GVHD. However, our metabolic studies could not detect any similarities between these nine patients and the acute GVHD patients; this makes the last explanation less likely.

Pretransplant characteristics are important for the risk of posttransplant acute GVHD, e.g. advanced disease, the conditioning regimens and previous viral infections (Chaidos et al. 2007; Gratwohl 2012). However, several studies have investigated the associations between serum or plasma biomarker levels and the risk of posttransplant complications, including acute GVHD. Firstly, the risk of serious complications early after allotransplantation is associated with the preconditioning serum profile of 
Fig. 4 Pathway enrichment analysis of metabolic profiles associated with an ongoing pretransplant acute phase reaction. The pathway enrichment analysis was used to identify metabolites/pathways that were altered in pretransplant samples derived from patients with an ongoing pretransplant acute phase reaction (i.e. serum CRP $>10 \mathrm{mg} / \mathrm{L}$ ) compared to patients with normal CRP level. A pathway enrichment value greater than 1 indicates that the pathway was increased in patients with an acute phase reaction. The top ranked metabolic pathways $(p<0.05$, enrichment value $>2$ ) identified by this comparison are given in the figure

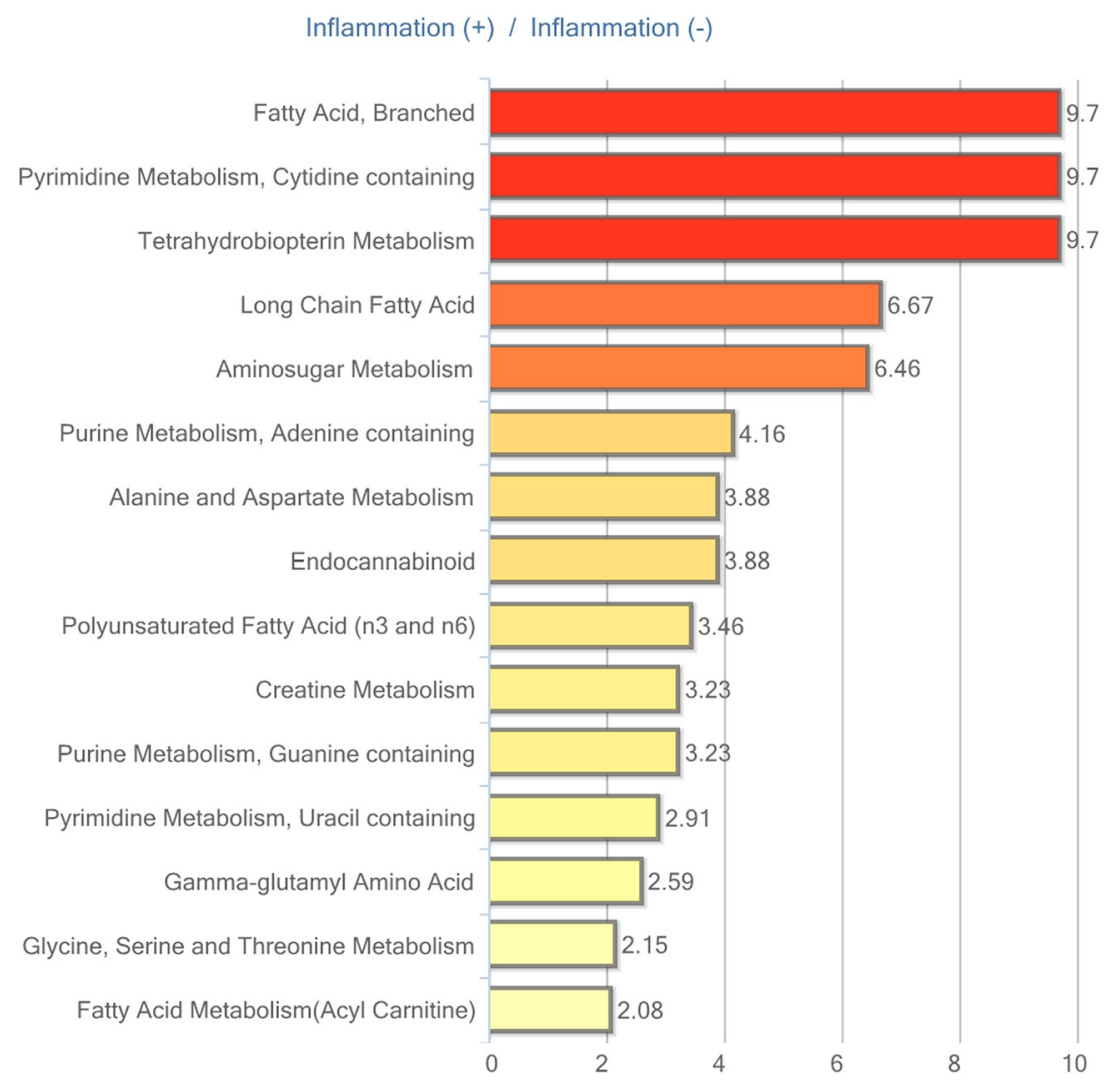

immunoregulatory and angioregulatory mediators, and the same seems to be true for systemic levels of endothelial cellderived adhesion molecules (Lindås et al. 2014; Reikvam et al. 2012a). Secondly, an association between serum levels of angioregulatory Angiopoietin-2/Tie2 and relapse-free survival was described for a selected group of high-risk patients with only 17 out of 90 patients being in complete remission prior to transplantation (Koenecke et al. 2010; Kümpers et al. 2008). These patients are thus not comparable to our unselected patients. Thirdly, in a pediatric study Porkholm et al. (Porkholm et al. 2013), described that pretransplant (but postconditioning) high Angiopoietin-2 plasma levels were associated with increased risk of gastrointestinal GVHD and non-relapse mortality. Thus, our present study is the first to suggest an association between the preconditioning metabolic profile and risk of later acute GVHD.

Tyrosine is synthesized from the essential amino acid phenylalanine and can be fermented in the gut, one important product being p-Cresol (de Loor et al. 2005). Phenols can also be formed by gut fermentation, including phenyl-propionic acid (Nordlund et al. 2012). A majority of our patients had acute leukemia in complete remission and had been treated with intensive antileukemic chemotherapy prior to the transplantation; this treatment causes mucositis and may cause severe gastrointestinal complications (Shafi and Bresalier 2010), and the production of p-cresols and phenols depends on the intestinal microenvironment, including intestinal flora and pH (Nakabayashi et al. 2011). The altered levels of such metabolites may thus reflect disturbances in the gastrointestinal function or the intestinal flora that are associated with a risk of acute GVHD. These metabolites can originate from bacterial fermentation in the large intestine (de Loor et al. 2005; Nordlund et al. 2012); their altered pretransplant levels may then predispose to acute GVHD through effects on donor immunocompetent cells.

$\mathrm{p}$-Cresol is absorbed from the gut and detoxified in the liver by conjugation (sulfatation and glucuronidation), the main product in serum being p-cresylsulfate and p-cresylglucuronide (de Loor et al. 2005). However, free p-cresol can also be detected in the circulation (Bammens et al. 2006; de Loor et al. 2005; Meijers et al. 2010). p-cresyl sulfate (and also p-cresyl glucuronide) is regarded as a proinflammatory mediator (Poveda et al. 2014); it activates leukocyte free radical production, has proapoptotic effects (Poveda et al. 2014) and alters the endothelial response to inflammatory cytokines as well as the cytokine release by monocytes (Schepers et al. 2007). Thus, these metabolites can possibly alter inflammatory processes both by direct effects on immunocompetent cells and by altered cell trafficking/migration due to altered endothelial cell functions. 


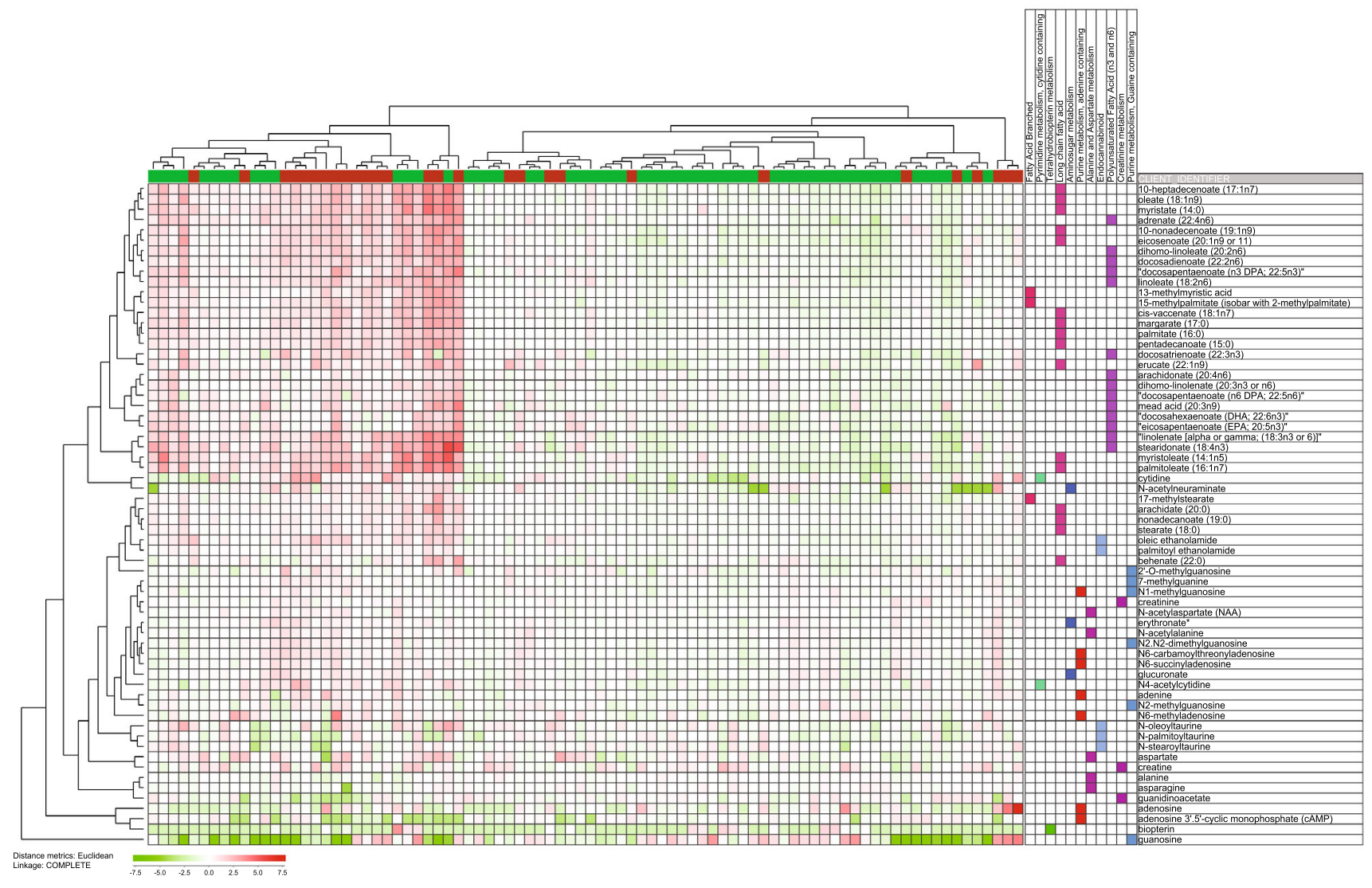

Fig. 5 Hierarchical clustering analysis identified two patient subset highly associated with pretransplant inflammation. The hierarchical clustering analysis (Pearsons Correaltion, complete linkage) was based on the 64 metabolites that belonged to the 11 terms/those metabolic pathways showing enrichment score $>3.0$ and $p<0.001$. The heat map together with dendrograms is presented in the figure. Red indicates high value and green low value, and the metabolic

Carnitine is an essential dietary nutrient and it acts as a carrier of fatty acids across the inner membrane of the mitochondrial membrane (Malaguarnera 2012). Oral propyonyl-L-carnitine has been tried in the treatment of human diseases (Andreozzi 2009; Malaguarnera 2012), mainly cardiovascular disease, but it has also shown beneficial effects in a randomized, double-blind, placebo-controlled study of patients with ulcerative colitis, i.e. an autoimmune disease (Mikhailova et al. 2011). The vascular effects of propyonylL-isocarnitine include reduced plasma extravasation, modulation of endothelin-prostaglandin effects, vasodilatation, and increased fatty acid oxidation in vascular tissue. These effects may then affect the trafficking of immunocompetent cells and thereby also the risk of acute GVHD.

Both in vitro and in vivo studies suggest that the branched chain amino acids have immunoregulatory effects that at least partly are mediated through mTOR activation (Kim et al. 2012). Firstly, leucine has been shown to increase the release of leptin in animal models (Lynch et al. 2006), another important metabolic immunoregulator (Agrawal pathways are shown to the left. We identified two main clusters; one including several patients with an acute phase reaction (left) and another including few of these patients (right). These two main clusters differed significantly with regard to the frequency of pretransplantation inflammation processes (Chi Square test, $\left.\chi^{2}=8.02, p=0.0046\right)$

et al. 2011). Secondly, especially valine seems important for the differentiation of normal monocytes to specialized and mature antigen-presenting dendritic cells, and this differentiation is then associated with a PI3K-Akt-mTOR induced metabolic switch to glycolysis (Kakazu et al. 2007). Finally, both leucine, isoleucine or valine seem to enhance the proliferation of activated $\mathrm{T}$ cells (Calder 2006), and animal studies suggest that oral intake of isoleucine has an immunoregulatory function in the gut (Zhao et al. 2014). Finally, several studies suggest that BCAA have immunostimulatory effects not only in animal models but also in humans (Alam et al. 2011; Bassit et al. 2002; Cerra et al. 1984; Freund et al. 1978; Nuwer et al. 1983). Thus, our study suggests that the pretransplant status of the immunoregulatory system is important for the risk of posttransplant acute GVHD.

We also analysed the metabolites associated with a pretransplant acute phase reaction (i.e. increased serum CRP levels), but the metabolic terms as well as individual metabolites associated with increased CRP levels differed 
from the terms/metabolites associated with acute GVHD. The same was true for terms/metabolites associated with early hematopoietic reconstitution. These observations suggest that the metabolic mechanisms associated with the risk of acute GVHD are independent of the acute phase reaction and bone marrow recovery.

\section{Concluding remarks}

Our present study suggests that the pretransplant metabolic profile reflects the risk of developing acute GVHD after allotransplantation. Several of the metabolites or the metabolic pathways that were observed to reflect this risk (tyrosine metabolites, BCAAs, fatty acid metabolism), are important for immunoregulation and may thus be directly involved in the development of acute GVHD through effects on donor immunocompetent cells. The question whether pretransplant metabolic interventions (i.e. nutritional support) will decrease the risk of posttransplant acute GVHD has to be addressed in future clinical studies.

\section{Compliance with ethical standards}

Conflict of Interest All authors declare that they have no conflicts of interest related to this manuscript.

Ethical approval All procedures performed in studies involving human participants were in accordance with the ethical standards of the institutional and/or national research committee and with the 1964 Helsinki declaration and its later amendments or comparable ethical standards.

Funding The study was supported by Øyvinn Mølbach-Pedersen's foundation, Helse Vest and the Norwegian Cancer Society.

Informed consent Informed consent was obtained from all individual participants included in the study.

\section{References}

Agrawal, S., Gollapudi, S., Su, H., \& Gupta, S. (2011). Leptin activates human B cells to secrete TNF-alpha, IL-6, and IL-10 via JAK2/STAT3 and p38MAPK/ERK1/2 signaling pathway. Journal of Clinical Immunology, 31, 472-478. doi:10.1007/ s10875-010-9507-1.

Alam, N. H., et al. (2011). L-isoleucine-supplemented oral rehydration solution in the treatment of acute diarrhoea in children: A randomized controlled trial. Journal of Health, Population, and Nutrition, 29, 183-190.

Andreozzi, G. M. (2009). Propionyl 1-carnitine: Intermittent claudication and peripheral arterial disease. Expert Opinion on Pharmacotherapy, 10, 2697-2707. doi:10.1517/ 14656560903215871.

Bammens, B., Evenepoel, P., Keuleers, H., Verbeke, K., \& Vanrenterghem, Y. (2006). Free serum concentrations of the proteinbound retention solute p-cresol predict mortality in hemodialysis patients. Kidney International, 69, 1081-1087. doi:10.1038/sj.ki. 5000115.

Bassit, R. A., et al. (2002). Branched-chain amino acid supplementation and the immune response of long-distance athletes. Nutrition, 18, 376-379.

Calder, P. C. (2006). Branched-chain amino acids and immunity. Journal of Nutrition, 136, 288S-293S.

Cerra, F. B., et al. (1984). Branched chain metabolic support. A prospective, randomized, double-blind trial in surgical stress. Annals of Surgery, 199, 286-291.

Chaidos, A., Kanfer, E., \& Apperley, J. F. (2007). Risk assessment in haemotopoietic stem cell transplantation: Disease and disease stage. Best Practice \& Research Clinical Haematology, 20, 125-154. doi:10.1016/j.beha.2006.10.003.

Chen, W. L., et al. (2014). A distinct glucose metabolism signature of acute myeloid leukemia with prognostic value. Blood, . doi:10. 1182/blood-2014-02-554204.

de Loor, H., Bammens, B., Evenepoel, P., De Preter, V., \& Verbeke, K. (2005). Gas chromatographic-mass spectrometric analysis for measurement of p-cresol and its conjugated metabolites in uremic and normal serum. Clinical Chemistry, 51, 1535-1538. doi:10.1373/clinchem.2005.050781.

Evans, A. M., et al. (2014). High resolution mass spectrometry improves data quanitity and quality as compared to unit mass resolution mass spectrometry in high-throghput profiling metabolomics. Metabolomics, 4, 132.

Filipovich, A. H. (2008). Diagnosis and manifestations of chronic graft-versus-host disease. Best Practice \& Research Clinical Haematology, 21, 251-257. doi:10.1016/j.beha.2008.02.008.

Freund, H. R., Ryan, J. A, Jr, \& Fischer, J. E. (1978). Amino acid derangements in patients with sepsis: Treatment with branched chain amino acid rich infusions. Annals of Surgery, 188, 423-430.

Gooley, T. A., et al. (2010). Reduced mortality after allogeneic hematopoietic-cell transplantation. New England Journal of Medicine, 363, 2091-2101. doi:10.1056/NEJMoa1004383.

Gratwohl, A. (2012). The EBMT risk score. Bone Marrow Transplantation, 47, 749-756. doi:10.1038/bmt.2011.110.

Kakazu, E., Kanno, N., Ueno, Y., \& Shimosegawa, T. (2007). Extracellular branched-chain amino acids, especially valine, regulate maturation and function of monocyte-derived dendritic cells. The Journal of Immunology, 179, 7137-7146.

Kersey, J. H. (2010). The role of allogeneic-cell transplantation in leukemia. New England Journal of Medicine, 363, 2158-2159. doi:10.1056/NEJMe1010818.

Kim, Y. M., et al. (2012). SH3BP4 is a negative regulator of amino acid-Rag GTPase-mTORC1 signaling. Molecular Cell, 46, 833-846. doi:10.1016/j.molcel.2012.04.007.

Koenecke, C., et al. (2010). Shedding of the endothelial receptor tyrosine kinase Tie 2 correlates with leukemic blast burden and outcome after allogeneic hematopoietic stem cell transplantation for AML. Annals of Hematology, 89, 459-467. doi:10.1007/ s00277-009-0869-5.

Kümpers, P., et al. (2008). Angiopoietin-2 predicts disease-free survival after allogeneic stem cell transplantation in patients with high-risk myeloid malignancies. Blood, 112, 2139-2148. doi:10. 1182/blood-2007-12-130021.

Lindås, R., Tvedt, T. H., Hatfield, K. J., Reikvam, H., \& Bruserud, O. (2014). Preconditioning serum levels of endothelial cell-derived molecules and the risk of posttransplant complications in patients treated with allogeneic stem cell transplantation. Journal of transplantation, 2014, 404096. doi:10.1155/2014/404096.

Lynch, C. J., Gern, B., Lloyd, C., Hutson, S. M., Eicher, R., \& Vary, T. C. (2006). Leucine in food mediates some of the postprandial rise in plasma leptin concentrations. American Journal of Physiology-Endocrinology and Metabolism, 291, E621-E630. doi:10.1152/ajpendo.00462.2005. 
Malaguarnera, M. (2012). Carnitine derivatives: Clinical usefulness. Current Opinion in Gastroenterology, 28, 166-176. doi:10.1097/ MOG.0b013e3283505a3b.

Meijers, B. K., et al. (2010). p-Cresol and cardiovascular risk in mildto-moderate kidney disease. Clinical Journal of the American Society of Nephrology, 5, 1182-1189. doi:10.2215/CJN. 07971109.

Mikhailova, T. L., et al. (2011). Randomised clinical trial: The efficacy and safety of propionyl-L-carnitine therapy in patients with ulcerative colitis receiving stable oral treatment. Alimentary Pharmacology \& Therapeutics, 34, 1088-1097. doi:10.1111/j. 1365-2036.2011.04844.x

Moura, A. P., Ribeiro, C. A., Zanatta, A., Busanello, E. N., Tonin, A. M., \& Wajner, M. (2012). 3-Methylcrotonylglycine disrupts mitochondrial energy homeostasis and inhibits synaptic $\mathrm{Na}(+)$, $\mathrm{K}(+)$-ATPase activity in brain of young rats. Cellular and Molecular Neurobiology, 32, 297-307. doi:10.1007/s10571-0119761-7.

Nakabayashi, I., et al. (2011). Effects of synbiotic treatment on serum level of p-cresol in haemodialysis patients: A preliminary study. Nephrology Dialysis Transplantation, 26, 1094-1098. doi:10. 1093/ndt/gfq624.

Nordlund, E., Aura, A. M., Mattila, I., Kosso, T., Rouau, X., \& Poutanen, K. (2012). Formation of phenolic microbial metabolites and short-chain fatty acids from rye, wheat, and oat bran and their fractions in the metabolical in vitro colon model. Journal of Agricultural and Food Chemistry, 60, 8134-8145. doi:10.1021/jf3008037.

Nuwer, N., Cerra, F. B., Shronts, E. P., Lysne, J., Teasley, K. M., \& Konstantinides, F. N. (1983). Does modified amino acid total parenteral nutrition alter immune-response in high level surgical stress. JPEN. Journal of Parenteral and Enteral Nutrition, 7, 521-524.

Porkholm, M., Bono, P., Saarinen-Pihkala, U. M., \& Kivivuori, S. M. (2013). Higher angiopoietin-2 and VEGF levels predict shorter EFS and increased non-relapse mortality after pediatric hematopoietic SCT. Bone Marrow Transplantation, 48, 50-55. doi:10.1038/bmt.2012.101.

Poveda, J., et al. (2014). p-cresyl sulphate has pro-inflammatory and cytotoxic actions on human proximal tubular epithelial cells. Nephrology Dialysis Transplantation, 29, 56-64. doi:10.1093/ $\mathrm{ndt} / \mathrm{gft} 367$.

Reikvam, H., Fredly, H., Kittang, A. O., \& Bruserud, O. (2013). The possible diagnostic and prognostic use of systemic chemokine profiles in clinical medicine-the experience in acute myeloid leukemia from disease development and diagnosis via conventional chemotherapy to allogeneic stem cell transplantation. Toxins, 5, 336-362. doi:10.3390/toxins5020336.
Reikvam, H., Hatfield, K. J., Fredly, H., Nepstad, I., Mosevoll, K. A., $\&$ Bruserud, O. (2012a). The angioregulatory cytokine network in human acute myeloid leukemia-From leukemogenesis via remission induction to stem cell transplantation. European Cytokine Network, 23, 140-153. doi:10.1684/ecn.2012.0322.

Reikvam, H., et al. (2012b). The pretransplantation serum cytokine profile in allogeneic stem cell recipients differs from healthy individuals, and various profiles are associated with different risks of posttransplantation complications. Biology of Blood and Marrow Transplantation, 18, 190-199. doi:10.1016/j.bbmt.2011. 10.007 .

Schepers, E., Meert, N., Glorieux, G., Goeman, J., Van der Eycken, J., \& Vanholder, R. (2007). P-cresylsulphate, the main in vivo metabolite of p-cresol, activates leucocyte free radical production. Nephrology Dialysis Transplantation, 22, 592-596. doi:10. 1093/ndt/gft584.

Servais, S., et al. (2014). Pre-transplant prognostic factors of longterm survival after allogeneic peripheral blood stem cell transplantation with matched related/unrelated donors. Haematologica, 99, 519-526. doi:10.3324/haematol.2013.089979.

Shafi, M. A., \& Bresalier, R. S. (2010). The gastrointestinal complications of oncologic therapy. Gastroenterology Clinics of North America, 39, 629-647. doi:10.1016/j.gtc.2010.08.004.

Sorror, M. (2009). Impacts of pretransplant comorbidities on allogeneic hematopoietic cell transplantation (HCT) outcomes. Biology of Blood and Marrow Transplantation, 15, 149-153. doi:10.1016/j.bbmt.2008.12.498.

Stavrum, A. K., Petersen, K., Jonassen, I., Dysvik, B. (2008). Analysis of gene-expression data using J-Express. Curr Protoc Bioinformatics Chapter 7, Unit 73 doi:10.1002/0471250953. bi0703s21.

Tvedt, T. H., Reikvam, H., Bruserud, O. (2016). Nutrition in allogenic stem cell transplantation-Clinical guidelines and immunobiological aspects. Current Pharmaceutical Biotechnology, 17, 92-104. doi:10.2174/138920101701151027163600.

Wang, J. H., et al. (2013). Prognostic significance of 2-hydroxyglutarate levels in acute myeloid leukemia in China. Proc Natl Acad Sci USA, 110, 17017-17022. doi:10.1073/pnas.1315558110.

Zatarain, L., \& Savani, B. N. (2012). The role of nutrition and effects on the cytokine milieu in allogeneic hematopoietic stem cell transplantation. Cellular Immunology, 276, 6-9. doi:10.1016/j. cellimm.2012.05.003.

Zhao, J., et al. (2014). Effect of dietary isoleucine on the immunity, antioxidant status, tight junctions and microflora in the intestine of juvenile Jian carp (Cyprinus carpio var. Jian). Fish \& Shellfish Immunology, 41, 663-673. doi:10.1016/j.fsi.2014.10.002. 